\title{
Bioclimatic generative design: energy optimizing skins
}

\author{
Santiago Saucedo \\ Universidad Nacional del Litoral | Argentina | santiagosaucedo01@gmail.com
}

María Elena Tosello

Universidad Nacional del Litoral | Argentina | maritosello@gmail.com

\begin{abstract}
Efficiency in the use of energy is one of the needs of contemporary times. Using the new instruments to bioclimatic design, opens up new possibilities. This study aimed to design generative processes that allow us to determine the best locations for different envelopes, through digital simulations related to a real environment. Being an investigation for design purposes, three types of actions were carried out: analyze, project and evaluate. The activities are developed from the logics of the parametric design; therefore, the processes are dynamic and allow variations, being able to be adjusted and repeated in any location in the world.
\end{abstract}

Keywords: Parametric design; Dynamic systems; Design processes; NURBS, Sustainable architecture

\section{INTRODUCCIÓN}

Estudios recientes demuestran que la construcción es la actividad que más contribuye al calentamiento global. Sin embargo, la relación entre la arquitectura y la eficiencia energética se puede mejorar para colaborar con el ecosistema. Como diseñadores en la era de las comunicaciones e Internet, es nuestra responsabilidad producir propuestas que promuevan una lógica de pensamiento y desarrollo sustentable. Si tenemos en cuenta el avance constante de la tecnología, podemos apreciar que el mundo está cambiando y nuestra disciplina, no puede ser la excepción. La arquitectura debe comprender y adaptarse a las nuevas circunstancias, utilizando los medios y generando aportes que ayuden a orientar los procesos de la cuarta revolución industrial.

El BP, Statistical Review of World Energy, es un estudio desarrollado por la compañía inglesa British Petroleum e indica que, teniendo en cuenta los consumos actuales, las reservas de petróleo se acabarán en el año 2067, por lo tanto, resulta evidente la necesidad de incorporar los sistemas de generación de energía natural a nuestro modelo de desarrollo.

Además, la temperatura del planeta aumenta todos los años a causa de los diferentes impactos que el hombre genera en su hábitat, y así, crece exponencialmente el consumo de energía con el fin de climatizar espacios interiores para lograr temperaturas de confort. Como diseñadores respetuosos y responsables del ambiente, es indispensable explorar acciones que, desde nuestra disciplina, den respuesta a las necesidades del ecosistema y de la sociedad.

El objetivo de nuestro estudio fue diseñar un proceso generativo capaz de crear pieles inteligentes y dinámicas, que capturen energía natural para los edificios, al mismo tiempo que los protejan de la radiación solar.

Como instrumento proyectual se utilizó el diseño generativo, un método basado en reglas matemáticas para analizar valores como temperatura, orientación del sol, cantidad de radiación emitida, fuerza del viento, etc., y generar resultados de diseño óptimos para un problema, a través de una solución única o de una familia de soluciones. Este sistema permite la generación de geometrías a partir de la definición de parámetros iniciales y la programación de las relaciones formales que guardan entre ellos.

El diseño generativo posibilita no sólo encontrar una solución a un problema determinado, sino hallar una familia de posibles soluciones para el problema, en forma ágil y precisa, simplemente modificando los parámetros del sistema desarrollado. De esta manera, se deben priorizar algunas variables sobre otras. Asimismo, la implementación de esta lógica permite que nuestro diseño pueda ser reinterpretado por el sistema para distintos casos.

\section{METODOLOGÍA}

Por tratarse de una investigación proyectual, se realizan tres tipos de acciones: analizar/diagnosticar, planificar/diseñar, y evaluar/reflexionar. A su vez, como el proceso de investigación es continuo, abierto y flexible, éste propone una dialéctica entre las actividades, las cuales no se desarrollan de manera lineal, sino a través de una espiral de carácter pro-alimentador que genera un movimiento constante entre el pensamiento y las prácticas, es decir, que todas las actividades y momentos del proceso están relacionados y constituyen una fuente de datos.

La fase de análisis y reconocimiento establece la definición del problema de estudio; el análisis de sus efectos sobre los sujetos y las prácticas; y su interrelación con otros problemas; la descripción de las condicionantes contextuales; y el reconocimiento de los saberes e instrumentos disponibles para estudiar alternativas de solución.

El análisis de los casos culmina con un diagnóstico reflexivo, esbozando un esquema relacional que vincula problemáticas, condiciones, instrumentos y posibilidades, y se expresa en anticipaciones de sentido o hipótesis 
La segunda etapa -diseño, planificación y proyecto- incluye actividades diversas que son interdependientes: planificar estrategias de diseño, acción y observación; modos y técnicas de evaluación pertinentes; e instrumentos de construcción de datos relevantes, en relación al objeto de estudio. Además, algunos procesos requieren diseñar interfaces y artefactos que integren sensores, con el objetivo de relevar y registrar la información de las condiciones ambientales para luego producir diferentes tipos de acciones, según sea necesario en cada caso.

Finalmente, la etapa de evaluar y reflexionar, consiste en explorar relaciones entre los procesos proyectuales y los instrumentos y artefactos tecnológicos integrados a éstos, de modo que pueden surgir relaciones nuevas y aplicables a la investigación desde la multidisciplina. Se efectúan verificaciones de funcionamiento y rendimiento para analizar el comportamiento de los indicadores en relación a estándares de control, a través de modelos o simulaciones, y experimentaciones con ejercicios de proyecto en cursos y talleres. La construcción de los datos relevantes requiere una triangulación de técnicas como observaciones, entrevistas y encuestas.

Luego se sistematizan, analizan e interpretan esos datos a fin de verificar si las propuestas responden o no a las premisas planteadas, así como también, sugerir influencias entre los distintos aspectos: diseño, tecnologías, materiales, usos y significados.

\section{DISEÑO EMERGENTE}

Todos los procesos se desarrollan a través de lógicas de diseño paramétrico generativo con el software Rhinoceros y el plugin Grasshopper. El diseño paramétrico es la abstracción de una idea o concepto, relacionada con procesos geométricos y matemáticos que nos permiten controlar con mayor precisión nuestro diseño para llegar a resultados más eficientes y adaptables. A su vez, el diseño generativo propone priorizar variables para obtener resultados optimizados, según categorías de análisis.

Se constituye como una alternativa al modelo lineal de diseño de los típicos softwares CAD, ya que permite relacionar todos los componentes del diseño y utilizarlos como variables modificables en tiempo real (Fig. 1).

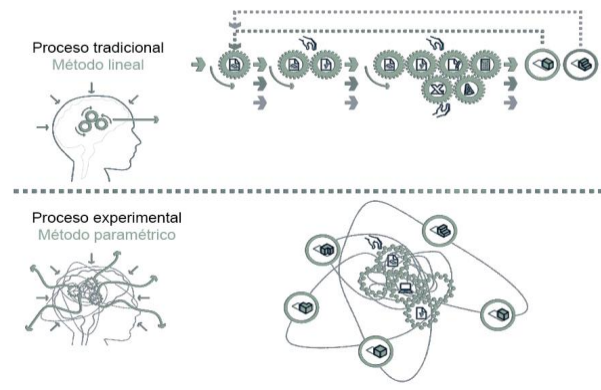

Figura 1: Comparación proceso tradicional y experimental. Fuente: Tesis de maestría Dalla Costa (2014).
El diseño paramétrico permite la generación de geometrías a partir de la definición de parámetros iniciales y la programación de las relaciones formales que guardan entre ellos, lo que posibilita no sólo encontrar una solución a un problema determinado, sino hallar una familia de soluciones posibles para el mismo problema de manera ágil y precisa, simplemente modificando los valores de los parámetros dentro del sistema desarrollado.

\section{RESULTADOS}

\section{SOBRE EL SECTOR}

Al implementar la parametrización en todos los aspectos de la investigación, resulta posible que nuestro diseño pueda ser reinterpretado por el sistema en distintos casos, según el lugar geográfico donde se ubicará el espacio que determinará la envolvente. Con el fin de lograr una mejor aproximación desde las lógicas paramétricas y posibilitar la realización de experimentaciones, se estableció un sector de estudio basándose en características propias de una ciudad de escala media. La región utilizada como área de estudio es la ciudad de Santa Fe y sus alrededores, delimitando una serie de condiciones climatológicas. El Aeropuerto Metropolitano de Santa Fe (AMSF) constituyó la fuente de datos climatológicos.

Los datos obtenidos y promediados por la estación en los últimos diez años fueron: humedad, temperatura de bulbo seco, temperatura de bulbo húmedo, dirección y velocidad del viento, radiación solar horizontal, radiación solar difusa, iluminancia global, iluminancia difusa, cobertura del cielo y presión atmosférica. Los datos se introdujeron a Grasshopper a través del complemento open-source "Lady Bug + Honey Bee", que son extensiones disponibles para Rhinoceros que sirven para analizar datos a fin de obtener resultados precisos y mapearlos de manera simple en el modelador 3D.

Primeramente, se realizó una verificación y contrastación entre los datos obtenidos del AMSF y las fuentes nacionales e internacionales disponibles que exponen sus datos de manera abierta. Dichas fuentes fueron: el Sistema Nacional de Datos Climáticos (SNDC, Argentina), donde la gestión fue facilitada por la fundación "Colectando Sol", y el National Aeronautics and Space Administration (NASA) Solar Data Deparment dependiente de los Estados Unidos, donde el ingreso a los datos es libre y gratuito. La comparación de la información determinó que los datos del AMSF son confiables y por lo tanto se decidió continuar con los mismos.

Para poder utilizar los complementos del plugin Grasshopper, fue necesario utilizar otros softwares que permiten la recolección de datos y mostrarlos de manera desencriptada, estos programas son: Open Studio, Energy Plus, Daysim, Radiance, entre otros (Fig. 2). A través del software Energy Plus, se pueden abrir archivos en formato .EPW (Energy Plus Weather Data File), un formato desarrollado por el U.S. Department of Energy (Departamento de Energía de los Estados Unidos), que almacena datos climatológicos. A su vez, el sitio web http://climate.onebuilding.org/ permite el acceso libre a archivos en formato.EPW donde se recopilan los datos de 
fuentes que cuentan con equipos de sensado certificados y se encuentran distribuidos alrededor del mundo.

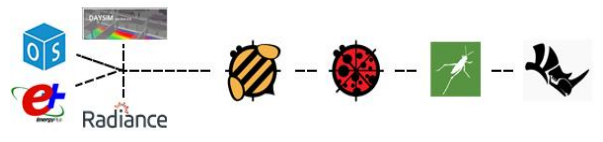

Figura 2: Diferentes softwares trabajan asociados a Honey Bee y se los incorpora a Grasshopper a través de Lady Bug, finalmente son visualizados en Rhinoceros.

\section{APROXIMACIÓN PARAMÉTRICA A LAS ENERGÍAS RENOVABLES}

Una vez obtenidos los datos, el siguiente paso fue determinar qué tipo de energía renovable deberían generar los módulos que conformasen la envolvente, por lo tanto, se recurrió nuevamente a las fuentes anteriormente mencionadas. Se delimitó el análisis a la energía solar y la energía eólica como motores de generación. Se analizaron lugares donde esas fuentes de energía se utilizan de manera masiva, determinando su alta eficacia. En segundo lugar, se compararon los datos del sitio elegido con los del sector de estudio previamente establecido, y finalmente, se optó por el sistema de generación solar.

El sitio de comparación elegido fue la ciudad de Los Ángeles, California, Estados Unidos. Según la California Energy Commission el $67 \%$ de la energía utilizada proviene de fuentes renovables, particularmente de energía eólica y energía solar, lo que constituye a esa ciudad como la de mayor generación de energía renovable en todo el continente americano.

Una vez seleccionado el sitio se repitió el proceso de obtención y verificación de datos climáticos. Esta vez los datos se obtuvieron de la University of California Los Angeles.

En primer lugar, se comparó la fuerza y velocidad del viento entre los dos lugares. Los resultados indican que la región de la ciudad de Santa Fe cuenta con la intensidad de vientos para alimentar un generador eólico, sin embargo, la dispersión del viento y los constantes cambios de dirección dificultarían generar energía eficazmente a lo largo de todo el año.

Finalmente, el estudio comparativo de radiación solar se realizó estableciendo como período de análisis el año 2019 (Fig. 3), y los resultados determinaron que la región de Santa Fe tiene las mismas potencialidades para la captación de energía solar, tomando en cuenta el promedio de potencia y constancia de radiación solar.

Es importante destacar que el estudio fue realizado íntegramente desde lógicas paramétricas, por lo tanto, replicar el estudio cambiando los factores sería una tarea que se puede desarrollar rápidamente. Así, el experimento queda abierto a la reinterpretación y adaptación a diferentes datos.

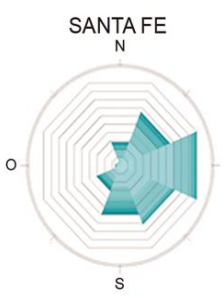

SANTA FE

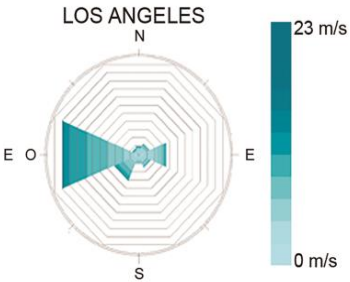

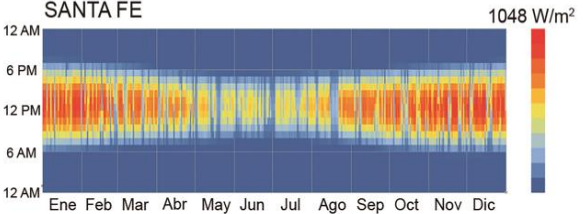

LOSANGELES

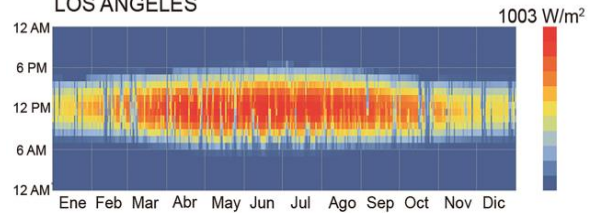

Figura 3: Estudios comparativos de viento y radiación solar. Gráfico de autor.

\section{ANÁLISIS GENERATIVO ARQUITECTÓNICO}

El funcionamiento del proceso generativo comienza con la etapa de análisis. En esta etapa se evalúa un espacio existente 0 a construir en un sector determinado, en nuestro caso utilizamos una forma compleja ubicada en un sector de la ciudad de Santa Fe, que nos permitió apreciar el comportamiento de la radiación solar en la pieza de estudio inmersa en un contexto específico, que arroja sombras y determina características diferentes a las que resultarían de analizar al objeto de manera aislada, es decir, sin involucrar a los edificios a su alrededor (Fig. 4).

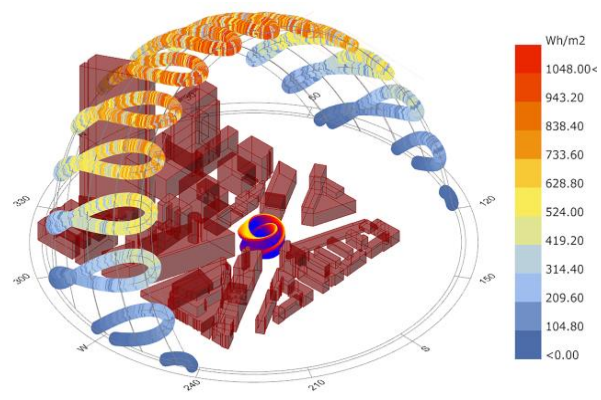

Figura 4: Comportamiento de la radiación solar sobre una superficie compleja en un entorno urbano.

Podemos apreciar entonces, las distintas posiciones del sol en los diferentes momentos del año y como irradian sobre la pieza analizada, de esta manera observamos el grado de precisión que determinan los programas y que posibilitan tener un control sobre el estudio. Una vez finalizado el mapeo sobre el espacio, se procedió a determinar cuáles serían las zonas que nos resultarían 
aprovechables para la captación de sol a través de paneles fotovoltaicos. Se procedió a realizar un estudio basado en el cálculo de la Hora Solar Pico (HSP), que luego se multiplica por la media alcanzada mensualmente a fin de obtener un valor de promedio solar anual que permita conocer cuanta energía estamos produciendo, cuanta necesitaría nuestro sistema para funcionar correctamente, y cuanta podríamos brindar al edificio, o en caso de ser una conexión Grid ON, cuanta podemos enviar a nuestro servidor de electricidad para vender como prosumidores.

Para obtener la HSP el cálculo es el siguiente: $\mathrm{Pi}$ * $\mathrm{D}$ * $\mathrm{RP}$. La sigla Pi significa potencia instalada (en nuestro caso tomaremos $1 \mathrm{kWp}$ [un kilowatt potencia] que es un valor estándar determinado para una potencia de uso no industrial); $D$ hace referencia a la cantidad de días (en este caso se utiliza un rango mensual y el estudio se repite todos los meses en todos los años que son analizados), y se toma como media 30 días del mes; y finalmente el valor RP refiere a la eficiencia del panel solar (se estipula que, según el modelo de panel solar, la eficiencia es aproximadamente el $75 \%$ del total posible de captación). A través del cálculo, se determina desde que valor de radiación solar los colectores obtendrán la mayor cantidad de energía posible, a modo de aprovechar el sol de manera eficaz y absorbiendo costos de manera rápida. Una vez conseguido este valor, se delimita la selección que resultará útil, y como el proceso está completamente parametrizado, los valores se pueden cambiar en cualquier momento si así lo precisa el diseñador. El siguiente paso es geometrizar la superficie según el tamaño del objeto para lograr un resultado proporcionado entre la cantidad de módulos que ocupará la envolvente y el espacio físico con que cuenta el objeto estudiado. Continuando con la lógica de diseño paramétrico, se procede a obtener el área y el centroide de cada una de esas secciones (Fig. 5). Esos puntos nos servirán para ubicar los módulos diseñados, combinando así los dos procesos paramétricos desarrollados independientemente el uno del otro, pero que resultan complementarios.

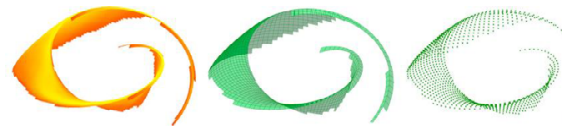

Figura 5: Seccionamiento, panelación y determinación de centroides. Gráficos de autor.

Hasta el momento conseguimos realizar el análisis del espacio que se pretende cubrir con la piel, un análisis detallado de la radiación, seccionar según cálculo de eficiencia y determinar los centros para ubicar los módulos. A continuación, introduciremos brevemente algunas obras que fueron tomadas como casos de estudio para ilustrar cómo se desarrollan e implementan pieles responsivas en diseños contemporáneos.

Las obras son de distintas escalas, lo que permite comprender mejor cómo se piensan estas envolventes desde su ideación hasta su fabricación. El Aegis Hyposurface (Fig. 6) es un diseño de los arquitectos de la firma dECOi. Se trata de una envolvente automatizada que responde a la proximidad de objetivos y al movimiento de los mismos. La obra se conforma a partir de módulos triangulados que, por sus características formas y materiales, representan un desafío para nuestra propia investigación, desde la dimensión de la morfología dinámica. El movimiento fluido de orden decreciente simula una ola expandiéndose sobre la piel, por lo tanto, también se entiende que el movimiento mismo fue diseñado y no es el efecto obtenido simplemente por la programación de los sistemas de input en relación con la activación de motores que generan el movimiento.

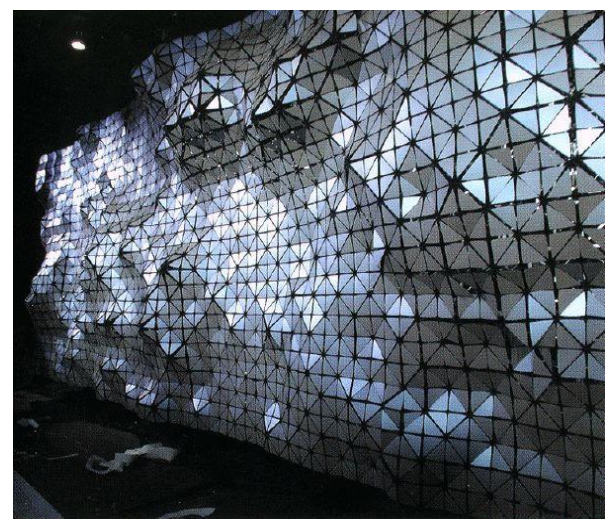

Figura 6: Aegis Hyporsurface. dECOi

Moloney explica que el concepto de indeterminación y pliegues tiene el objetivo de combinar imagen y forma, creando una nueva "zona arquitectónica" a la que determina el flujo constante. La siguiente obra estudiada es el conjunto de las torres Al Bahar (Fig. 7) ubicadas en la ciudad de Abu Dhabi, capital de los Emiratos Árabes Unidos. Las mismas fueron diseñadas y construidas por la firma Aedas, consagrándose ganadora de un concurso internacional donde los principales ejes proyectuales eran el diseño ambientalmente consciente y la cultura local. En ellas funciona la sede del Consejo de Inversiones de Abu Dhabi y son parte de un plan de desarrollo llamado 2030 Abu Dhabi. Las torres cuentan con pieles móviles que se abren y se cierran conformando un sistema de sombreado árabe tradicional llamado Masharabiya, el cual fue reinterpretado por el equipo de arquitectos informáticos de la firma Aedas. Éstos lograron establecer las fórmulas matemáticas necesarias para poder parametrizar el diseño de las envolventes y, a su vez, realizar simulaciones de comportamiento dinámico según las características climáticas, precisando la ubicación geográfica de las torres.

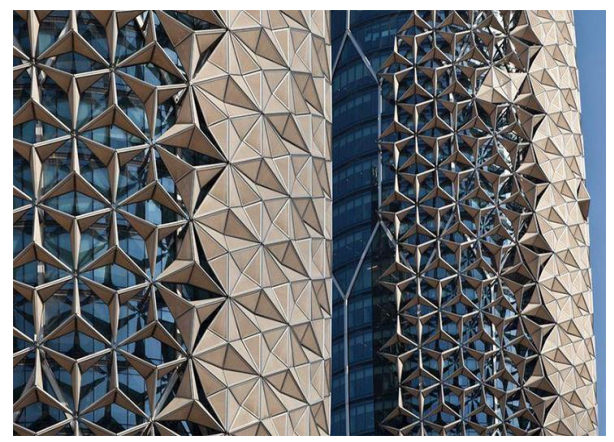

Figura 7: Envolventes Torres Al Bahar, Aedas. 
Para lograr resultados eficaces, Aedas se asoció con la firma ingenieril Arup, ambas partes trabajaron en equipo con el objetivo de perfeccionar los resultados obtenidos en las simulaciones. Los estudios indican que las pieles reducen la carga de calor ofreciendo ahorrar hasta un $20 \%$ de energía al total del conjunto, dato aún más sorprendente por encontrarse el complejo en el desierto.

La última obra analizada es el estadio Showcase (Fig. 8) de la firma Ove Arup Architects, ubicado en la ciudad de Doha, Qatar. El proyecto fue diseñado pensando en la FIFA World Cup Qatar 2022 (Copa Mundial de la FIFA, Qatar 2022), y pretende demostrar cómo arquitectura, ingeniería y tecnologías de vanguardia pueden combinarse para refrigerar los estadios sede del evento deportivo. De ahí el nombre de la obra: "estadio muestra". El Showcase no sólo refrigera el ambiente, sino que en su construcción se realizaron cero emisiones de carbono, lo cual lo convierte en un modelo de diseño y ejecución en el campo de la arquitectura sustentable. El mismo cuenta con una cubierta doble conformada por dos cuartos de elipsoides superpuestos, donde la superficie inferior rota de acuerdo a la orientación solar disminuyendo la radiación recibida en el campo de juego y en el sector de espectadores. Los techos se encuentran configurados por una serie de patrones triangulares que conforman un ritmo geométrico y generan lo que los autores de la obra llaman "la nube", por la obstrucción solar que provoca. Además, el techo se puede manipular permitiendo controlar los fuertes vientos que azotan la ciudad de Doha. Según los especialistas de la FIFA, el estadio demoró 2 horas en pasar de los $44^{\circ}$ a los $23^{\circ}$ deseados. Además, el mismo cuenta con una "granja solar", un concepto que no sólo involucra paneles fotovoltaicos, sino que también utiliza espejos parabólicos para concentrar la potencia de la energía solar.

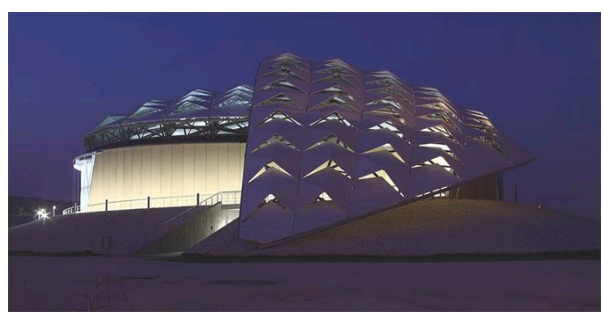

Figura 8: Showcase Stadium, Ove Arup Architects

En base a lo analizado en las obras, se puede observar que el mundo ha tomado conciencia sobre la importancia de construir pensando en la ecología. La incorporación de la tecnología en la conformación de las envolventes es una tendencia que se produce en todas las construcciones que han optado por plantear diseños estratégicos, no sólo respecto al confort, sino también buscando recopilar datos a través de medios mensurables y estadísticos como ahorro de energía, emisión de gases y fases de proyección y ejecución de obra. A su vez, se determina que el factor estético no es un mero valor agregado, por el contrario, es pensado con el mismo detenimiento que la ubicación geográfica y los datos climatológicos. Los movimientos generan una nueva posibilidad desde la dimensión de la percepción visual del público, y merecen un lugar importante en la fase de diseño previa a la ejecución. Otra consideración a destacar, es la magnitud que ocupa el valor simbólico de la ubicación, pero también del contexto social y cultural.

Si bien los análisis climatológicos pueden resultar interesantes para tomar decisiones, se debe tener en cuenta el contexto socio-económico del lugar donde se planea llevar adelante un proyecto, indistintamente de la escala del mismo. A modo de cierre de la etapa de estudio y reflexión de los antecedentes, se hace una valoración concluyendo que diseñar en función del clima y la tecnología puede ser igual o superador a un diseño convencional, además, pensando en el futuro de nuestra profesión y del medioambiente, no sólo es innovador proyectar desde el diseño bioclimático, sino que también es necesario.

\section{GENERACIÓN DEL PROTOTIPO}

Lo primero a tener en cuenta al momento de desarrollar el diseño del módulo, son los elementos que debe ser capaz de incorporar, entre ellos se destacan la placa Arduino, la célula solar estándar (se pondera la utilización de elementos estándar para disminuir costos de producción, por lo tanto, los valores volumétricos de los mismos son fijos y no variables dentro de la secuencia generativa), los mecanismos motrices sencillos (servomotores) y una batería de ciclo profundo de baja capacidad.

Por lo tanto, el dispositivo se diseñó incluyendo un panel fotovoltaico estándar de $250 \mathrm{~W}$, sin embargo, se lo toma a modo de prototipo ya que los paneles fotovoltaicos se comercializan con distintas formas y medidas. El sostén de los módulos en el edificio, en caso de que se incluyan a una obra ya construida, deberá ser una estructura externa para soportar el peso propio y las interacciones del viento. Dicha estructura debe ser fijada a la estructura original del edificio y no generará variaciones significativas a las cargas de la misma.

En esta primera prueba, el módulo consta de 8 pantallas de elementos textiles, sujetas a una estructura metálica donde las mismas varían su posición según el momento del día y la estación del año (Fig. 9)
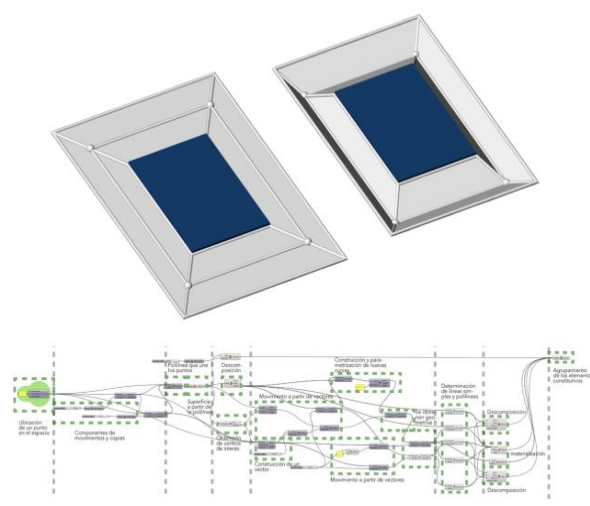

Figura 9: Generación paramétrica del módulo sencillo 
El módulo se abrirá en días cálidos para filtrar el ingreso del sol al edificio o se cerrará en estaciones frías para permitir un máximo calentamiento en el interior del espacio (Fig. 10). La orientación de los módulos no es arbitraria, ya que se encuentra directamente relacionada con el estudio de radiación que se explicó anteriormente. A su vez, la celda que se ubica en el medio del módulo se conecta a la batería donde la energía se almacena para que el dispositivo pueda moverse de manera autónoma y para el uso específico según la función, o para la venta energética desde la actividad de prosumidores.

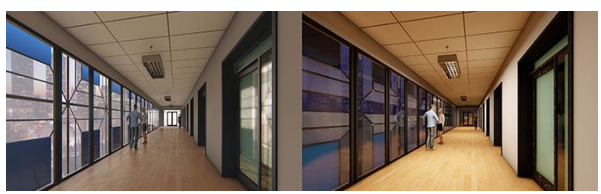

Figura 10: Visualización del módulo desde un interior en el día y en la noche.

Del mismo modo que la fase de análisis solar del cuerpo complejo, el diseño del módulo se basa en relaciones paramétricas que permiten no sólo llegar a una familia de resultados, sino también realizar simulaciones sencillas para comprobar el funcionamiento del dispositivo en su conjunto. Además, al encontrarse en relaciones variables, todos los componentes pueden variar sus tamaños sin representar un gran desafío proyectual, por ejemplo, para adaptar el dispositivo a distintas escalas según el edificio donde fuese a implementarse.

Como se explicó previamente, los módulos pueden dirigir la energía a grupos de baterías que funcionan conectadas, es decir, que se llena más de una, o la electricidad puede ir directamente a una red eléctrica disminuyendo el consumo energético de una residencia o inmueble. De ese modo, la empresa descuenta la electricidad aportada por los módulos, ahorrando energía y dinero al pagar los costos normales. Cuando el sistema envía la energía a la red, se trata de un sistema GRIN ON, en caso contrario es un sistema GRIN OFF. En asentamientos urbanos generalmente se recurre a la primera opción porque se ahorran gastos operativos y se necesitan menos elementos.

\section{DISCUSIÓN}

Podemos presumir que las pieles dinámicas no sólo permitirán ahorrar energía, sino que también la generarán pudiendo costear su propia construcción e instalación, especialmente en lugares donde la actividad de prosumidores está alentada por las empresas de electricidad. Además, quedó demostrado que todos los procesos hasta aquí realizados se han llevado adelante con lógicas paramétricas $y$, por lo tanto, todas las actividades pueden repetirse fácilmente en cualquier locación, ya que las bases de datos climáticas han sido testeadas y resultaron efectivas en comparación con las demás fuentes consultadas.

De este modo, se pueden ahorrar muchas horas de tedioso y repetitivo trabajo de análisis y, a la vez, se genera una familia de soluciones aplicables a cualquier espacio diseñado. Pensamos que las envolventes de un edificio no deben ser sólo protección y cerramiento, también deben ser capaces de generar energía e información, comunicar y modificarse.

La presente investigación no sólo diseña envolventes, sino también procesos conceptuales que se pueden continuar desarrollando y repitiendo a través de lógicas generativas para obtener resultados formales y teóricos, tanto a escala urbana como arquitectónica.

Las envolventes arquitectónicas diseñadas, promueven el uso de tecnologías de código abierto, atendiendo a la flexibilidad de programación desde las distintas locaciones, y desarrollos posteriores a partir de las mejoras promovidas por la comunidad open-source. La Dra. Arq. Camporeale (2017) explica en su tesis doctoral que la optimización basada en la performance energética, ha demostrado su potencial para integrar diseño y energía cuando se aplica a las primeras etapas del proceso de diseño o a la rehabilitación de un edificio existente.

Compartimos esta idea y pretendemos desarrollarla desde los sistemas emergentes. Por lo tanto, las líneas de acción a futuro son: explorar nuevas posibilidades para el diseño de los módulos desde la eficiencia, los materiales y la dimensión estética; construir suficientes prototipos funcionales de los módulos para ambientar un espacio; y poder estudiar los resultados obtenidos para luego compararlos con los arrojados por la simulación digital, a fin de verificar los mismos y determinar el nivel de viabilidad del sistema.

\section{REFERENCIAS}

Abdelmohsen, S.; Masud, P.; Elshafei, A. (2016). Using Tensegrity and Folding to Generate Soft Responsive Architectural Skins. eCAADe. (ecaade2016_210).

Camporeale, P. (2017). El uso de algoritmos genéticos aplicados al diseño bioambiental paramétrico. Universidad Nacional de La Plata, La Plata. Tesis doctoral.

California Energy Comission (2018). "La comisión de energía de California. Desarrollo de energías renovables" [en línea]. [Fecha de consulta: $1^{\circ}$ de Marzo de 2018]. Recuperado de: https://www.energy.ca.gov/releases/2018_releases/2018-0215 iepr2016 spanish nr.pdf

Chiarella M.; Raffin A.; Lopaczek S.; Martini S.; Góngora N.; Bressan F. (2014). Pieles Arquitectónicas Dinámicas. Prototipos a escala mediante prototipado rápido, microcontroladores y patrones plegados. SiGraDi. (sigradi2014_273)

Dalla Costa, M. (2014). Sistemas generativos dinámicos. Estrategias proyectuales paramétricas simples para prácticas arquitectónicas locales. Facultad de Arquitectura. UCC. Tesis de maestría.

Fraile, M. (2012). El Nuevo Paradigma Contemporáneo. Del Diseño Paramétrico a la Morfogénesis Digital. Teoría de la Arquitectura en la Contemporaneidad. Proyecto y Creación Científica en las Memorias Descriptivas. Buenos Aires: Universidad de Buenos Aires.

Gausa, M. (2001). Diccionario Metápolis de Arquitectura Avanzada. Barcelona: Actar.

Manovich, L. (2013). El software toma el mando. España. Ed. UOC.

Moloney, J. (2011). Designing kinetics for architectural facades: State change. Londres: Routledge.

Tosello, M. (2016). El espacio-interfaz del dispositivo hipermedial dinámico. Universidad Nacional de Rosario. Tesis doctoral. 